\title{
Étude des méthodes de calcul des pressions de contact dans les roulements à pistes intégrées des boîtes de transmission aéronautiques
}

\author{
Laurent Zamponi $^{1}$, Emmanuel Mermoz ${ }^{1}$ et Jean-Marc Linares ${ }^{2, a}$ \\ 1 Eurocopter, Aéroport International Marseille Provence, 13725 Marignane Cedex, France \\ ${ }^{2}$ CIME/EA(MS)2, Université de la Méditerranée, 2 Av. Gaston Berger, 13625 Aix en Provence Cedex 1, France
}

Reçu le 6 octobre 2006, accepté le 6 juin 2007

\begin{abstract}
Résumé - De plus en plus, les concepteurs utilisent des méthodes avancées de calcul, pour optimiser les performances des systèmes mécaniques. Ce constat est d'autant plus vrai dans le milieu aéronautique où le rapport poids puissance doit être le plus faible possible. Certains logiciels de CAO (CATIA, SOLIDWORKS, PRO ENGINEER, etc.) offrent la possibilité d'effectuer des calculs de dimensionnement. Néanmoins, ce type d'outil ne permet pas, à ce jour, d'estimer facilement les pressions de contact entre deux pièces. Cet article présente les différentes méthodologies utilisables dans un environnement CAO pour le calcul des pressions de contact dans les roulements à pistes intégrées de boîte de transmission aéronautique. Ces méthodologies sont analytiques (théorie de Hertz), numériques (éléments-finis) ou hybrides. Ces différentes approches sont analysées et comparées suivant les critères de précision, de temps de calcul et de leurs aptitudes à s'intégrer dans un processus de conception industriel. À partir de cette analyse, il sera proposé une méthodologie de calcul des pressions de contact.
\end{abstract}

Mots clés : Roulement / CAO / éléments-finis / théorie de Hertz / boîte de transmission

\begin{abstract}
Study of methodologies for contact pressure calculation in integrated raceways bearings for aeronautical gearbox. More and more designers use advanced calculation methodologies for the optimisation of mechanical part performance. This is even truer in the aeronautic industry, where the power weight ratio must be as low as possible. Some CAD softwares (CATIA, SOLIDWORKS, PRO ENGINEER, etc.) provide the possibility to do complex computation. However these kinds of tools aren't currently able to easily estimate the contact pressure between two parts. This paper deals with the various methodologies usable in a CAD environment for the calculation of contact pressures in integrated raceways bearings for aeronautic gearbox. These methodologies may be analytical (Hertz theory), numerical (F.E.M.) or hybrid. They are analyzed and compared according to their precision, calculation time and readiness to be integrated in the industrial design process. An optimum contact pressure calculation methodology will be presented based upon the results of this analysis.
\end{abstract}

Key words: Rolling bearing / CAD / finite-element method / Hertz theory / gearbox

\section{Introduction}

En conception mécanique, le roulement est l'une des pièces les plus fréquemment utilisées pour réaliser des liaisons entre sous-ensembles en rotation. La connaissance de son fonctionnement est essentielle pour la maîtrise de la conception et de la fiabilité des mécanismes. Les normes ISO ainsi que les catalogues des fabricants de roulements permettent au concepteur d'implanter ce composant dans

\footnotetext{
a Auteur pour correspondance : linares@univmed.fr
}

un mécanisme. Dans l'industrie aéronautique et, plus particulièrement, dans la construction d'hélicoptères, la recherche de la performance, en minimisant la masse embarquée, est essentielle. Une boîte de transmission de puissance, ayant comme caractéristique une masse de $300 \mathrm{~kg}$ et pouvant transmettre une puissance de plusieurs mégawatts, est présentée sur la figure 1. Dans ce type de boîte de transmission de puissance d'hélicoptère, le gain de masse est obtenu en minimisant le nombre et l'épaisseur des pièces. Pour le guidage en rotation par éléments roulants, le gain de masse s'obtient en 


\section{Nomenclature :}

\begin{tabular}{|c|c|}
\hline$F_{\mathrm{r}}, F_{\mathrm{a}}, M:$ & charges appliquées sur le roulement, respectivement, effort radial, effort axial et moment \\
\hline$Q_{i}:$ & effort normal de contact sur l'élément roulant $i$ \\
\hline & raideur globale de contact entre la bille et les pistes \\
\hline$(\mathrm{O}, X, Y, Z):$ & $\begin{array}{l}\text { repère cartésien, avec } \mathrm{O} \text { centre géométrique de la piste intérieure, } Z \text { colinéaire à l'axe de rotation, } \\
X \text { parallèle à la direction de l'effort radial, et } Y \text { complète le trièdre }\end{array}$ \\
\hline$\psi_{i}:$ & angle entre la bille $i$ et l'axe $X$ dans le plan $X Y$ \\
\hline $\mathrm{O}^{\prime}:$ & centre de piste intérieure après chargement \\
\hline$\left(\mathrm{O}, U_{i}, V_{i}, Z\right):$ & repère cartésien liée à la bille $i$, tel que $\psi_{i}=\left(X, U_{i}\right)=\left(Y, V_{i}\right)$ dans le plan $X Y$ \\
\hline$\alpha_{i}:$ & angle de contact de la bille $i$ dans le plan $U_{i} Z$ \\
\hline$\delta_{i}:$ & déformation globale de contact entre la bille $i$ et les bagues \\
\hline$\delta_{\mathrm{r}}:$ & déplacement radial relatif entre la bague intérieure et la bague extérieure \\
\hline$\delta_{\mathrm{a}}:$ & déplacement axial relatif entre la bague intérieure et la bague extérieure \\
\hline$\theta:$ & rotation relative entre la bague intérieure et la bague extérieure \\
\hline$J \quad:$ & jeu diamétral \\
\hline$d_{\mathrm{m}}:$ & diamètre moyen \\
\hline$d_{\mathrm{mi}}:$ & grand diamètre de la piste intérieure \\
\hline$d_{\mathrm{me}}:$ & grand diamètre de la piste extérieure \\
\hline$r_{\mathrm{i}}:$ & rayon de la piste intérieure \\
\hline$r_{\mathrm{e}}:$ & rayon de la piste extérieure \\
\hline$D:$ & diamètre des billes \\
\hline$A_{i}:$ & point intersection entre le grand cercle de la piste extérieure et le plan $U_{i} Z$ \\
\hline$B_{i}:$ & point intersection entre le grand cercle de la piste intérieure et le plan $U_{i} Z$ \\
\hline$\delta_{\mathrm{r}}^{\text {def }} ; \delta_{\mathrm{a}}^{\text {def }}:$ & $\begin{array}{l}\text { champ de déplacements issus de la déformation des pièces calculé par E.F., respectivement, } \\
\text { champ de déplacement radial, champ de déplacement axial }\end{array}$ \\
\hline$U_{\mathrm{ri}}^{p} ; U_{\mathrm{ai}}^{p}:$ & $\begin{array}{l}\text { déplacements entre la piste intérieure et extérieure au voisinage de l'élément roulant } i \text {, } \\
\text { respectivement, déplacement radial, déplacement axial }\end{array}$ \\
\hline$U_{\mathrm{ri}}^{* p} ; U_{\mathrm{ai}}^{* p}$ & $\begin{array}{l}\text { déplacements entre les zones d'accrochage de l'élément de substitution } i \text {, } \\
\text { respectivement, déplacement radial, déplacement axial }\end{array}$ \\
\hline$K_{i}^{p}:$ & raideur équivalente de l'élément de substitution $i$ au pas de calcul $p$ \\
\hline
\end{tabular}

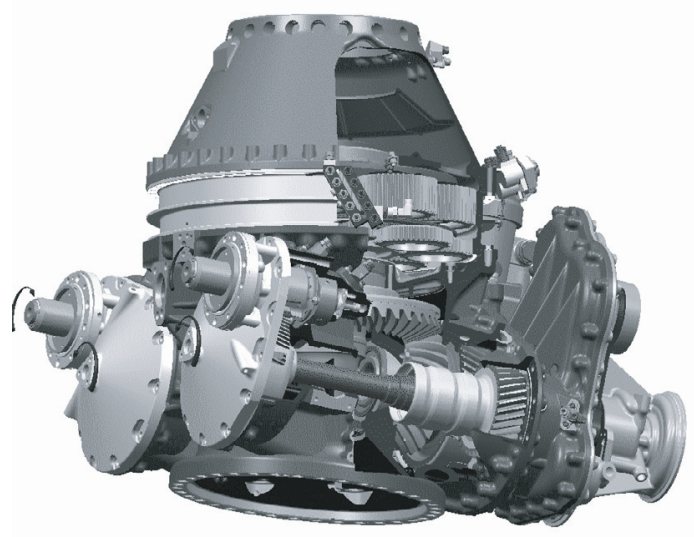

Fig. 1. Boîte de transmission principale d'hélicoptère vue en 3D.

remplaçant certaines bagues des roulements par des pistes intégrées sur les arbres ou sur les logements. Nous nommerons dans la suite de l'article, ce type de guidage par éléments roulants : roulement à pistes intégrées. Un pignon arbré équipé de roulements à pistes intégrées est présenté sur la figure 2. Ces pistes intégrées de formes cylindriques ou toriques sont fabriquées en interne par l'hélicoptériste. La qualité de la surface des pistes intégrées n'est pas, en toute rigueur, identique à

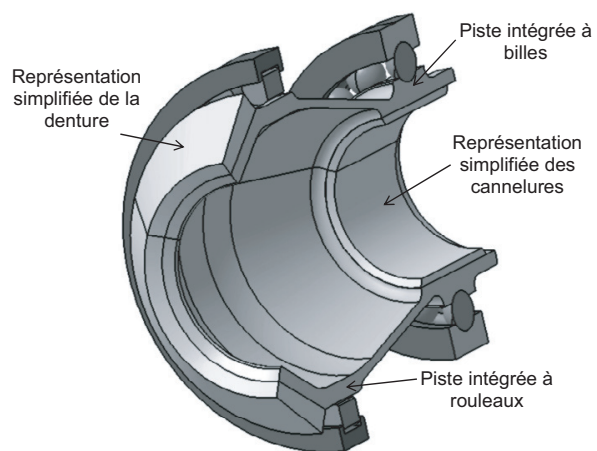

Fig. 2. Pignon arbré équipé de roulements à pistes intégrées.

celle obtenue par les roulementiers car la géométrie d'une pièce complexe impose des contraintes d'usinage bien plus grandes que celles obtenues sur une simple bague cylindrique. Dans ce cas, la méthode de dimensionnent classique sur la durée de vie des roulements ne peut pas être utilisée. La norme ISO 281 [1] stipule clairement qu'elle « n'est pas applicable à des constructions dans lesquelles les éléments roulants portent directement sur l'arbre ou dans un logement, à moins que leur surface ne soit à tous égards équivalente à celle du chemin de la bague ou de la rondelle qu'ils remplacent $»$. Pour dimensionner ces liaisons, il est nécessaire d'effectuer une modélisation 
complète du mécanisme afin de prendre en compte les raideurs des pièces, les jeux dans les roulements, les contacts, etc. Ce type de modélisation peut être partiellement effectué par les fabricants de roulements. Chaque fabricant de roulement a développé ses outils pour effectuer ce genre de modélisation. À l'heure actuelle, le dimensionnement des roulements à pistes intégrées des boîtes de transmission d'hélicoptère s'effectue en deux phases. Durant la première, le concepteur dimensionne le roulement à pistes intégrées comme un roulement avec la méthode de calcul de la durée de vie issue de l'ISO 286 [1] et des règles «métier $»$. Ensuite, la liaison pivot étudiée est envoyée chez le fabriquant de roulements pour la vérification du dimensionnement des roulements à pistes intégrées à l'aide de leurs outils de calculs.

Dans le but de minimiser les cycles de développement et d'optimiser globalement le mécanisme, il serait intéressant pour le concepteur d'avoir directement accès à ce type d'outils dans l'environnement CAO. Dans le secteur des mécaniques pour hélicoptère, les roulements à pistes intégrées des boîtes de transmission sont fortement chargés. De plus, l'expérience acquise par l'hélicoptériste et les roulementiers a permis de montrer que la pression de Hertz est un des critères qui impacte la fiabilité des pistes intégrées et leurs tenues en service. Un des critères de dimensionnement principal est la pression de contact entre les éléments roulants et la piste.

L'objectif général de nos travaux est de proposer une méthode de calcul des pressions de contact sur les pistes des roulements à pistes intégrées en incluant les déformations des pièces du mécanisme. Cette méthode devra s'intégrer dans un logiciel de CAO industriel. Dans cet article, la majorité des méthodes permettant d'obtenir les pressions de contact dans les roulements d'un mécanisme seront présentées. Nous étudierons si ces méthodes sont capables de prendre en compte les déformations des pièces et si elles sont intégrables dans un environnement CAO. Afin d'illustrer le déploiement de ces méthodes, applicable aux roulements usuels ou à pistes intégrées, l'exemple d'un roulement à billes sera utilisé.

\section{Bibliographie sur le dimensionnement des roulements}

Le professeur Stribeck a été l'un des premiers scientifiques à publier un travail sur la répartition des charges dans les roulements. Dans son rapport [2], il a proposé l'étude du contact bille-piste en corrélant la théorie de contact de Hertz à plusieurs séries d'essais. Il a obtenu une relation entre l'effort radial appliqué sur le roulement et l'effort sur l'élément roulant le plus chargé. Ces travaux sont à l'origine de la norme ISO 76 [3] sur le calcul de la charge statique de base. Lundberg et Palmgren [4] ont étudié le comportement en fatigue des roulements, ce qui a permis d'obtenir un critère de dimensionnement en fatigue. Ce travail a contribué à la mise en place de la norme ISO 281 [1]. Une évolution importante de la connaissance sur le comportement des roulements a été réalisée au milieu du XX $X^{\mathrm{e}}$ siècle par les travaux de Jones [5]. Ils portent sur l'étude de leur comportement statique et dynamique. Ces derniers ont été généralisés par Harris [6]. Ils sont la base de toutes les études théoriques des roulements.

La méthode proposée par Harris fait l'hypothèse que les déformations dans un roulement proviennent seulement des écrasements locaux dans les zones de contact. Cette hypothèse n'est plus valable si les bagues des roulements ont une épaisseur faible et d'autant plus si les pistes sont intégrées dans l'arbre ou le logement. Dans l'article de Harris et Jones [7], la méthode d'Harris a été modifiée en utilisant des coefficients de souplesse qui prennent en compte les déformations de la piste extérieure du roulement de satellite d'un train épicycloïdal. Cette piste a été intégrée sur le satellite juste sous la denture. Zupan et Prebil [8] ont généralisé cette approche en utilisant les éléments-finis pour calculer la matrice de souplesse de la structure afin de prendre en compte les déformations globales de la structure. Le calcul de la matrice de souplesse est limité par la taille des structures car l'inversion de la matrice de raideur demande des temps de calcul élevés. Hauswald et Houpert [9] et [10], pour pallier à ce problème, ont utilisé la technique de condensation de matrice (condensation de la matrice de raideur aux nœuds de liaisons). Bourdon et al. [11] ont développé un modèle hybride pour prendre en compte les déformations globales, où le mécanisme est discrétisé en éléments-finis et les éléments roulants de chaque roulement sont remplacés par des éléments-finis non-linéaires accrochés aux deux bagues. Les travaux de Lovell et al. [12] sur le contact entre une bille et deux plaques parallèles, modélisé par les éléments-finis ont montré que les résultats obtenus sont proches de ceux issus de la théorie de Hertz. Zhao [13] a utilisé un code éléments-finis 2D de contact, pour calculer les répartitions de charge dans un roulement soumis à un effort radial. Kang et al. [14] propose une modification de la loi de contact de Hertz par une loi issue d'une modélisation locale du contact par éléments-finis. Houpert [15], et Tanaka [16] ont proposé une amélioration analytique de la loi de Hertz pour les contacts dans les roulements. Antoine et al. [17] ont suggéré une formulation empirique et explicite de la loi de Hertz afin d'éviter la résolution numérique.

D'après la bibliographie, trois types de méthodes sont utilisés pour analyser les contacts dans les roulements. Elles sont analytiques, numériques ou hybrides. Pour les structures mécaniques et les technologies de roulements utilisées dans nos applications, il est nécessaire de prendre en compte la déformation de toutes les pièces. Généralement, sur les mécanismes étudiés, seulement $70 \%$ du déplacement relatif (hors jeux) entre les pistes intérieure et extérieure sont issus des déformations locales aux contacts entre les éléments roulants et les pistes, $20 \%$ du déplacement relatif proviennent des déformations de la piste extérieure et du carter, le reste, soit $10 \%$, est dû à la déformation de l'arbre (piste intérieure intégrée sur l'arbre). Dans les sections suivantes, certaines méthodologies proposées dans la bibliographie seront décrites, analysées et adaptées aux contraintes des 


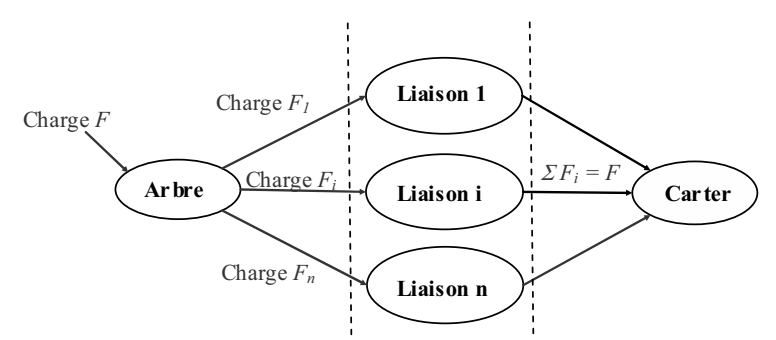

Fig. 3. Diagramme de transmission des efforts dans un mécanisme simple.

roulements à pistes intégrées dans les boîtes de transmission d'hélicoptères.

\section{3 Études des différentes méthodes}

\subsection{Problématique physique}

Dans l'étude des mécanismes, le calcul des charges aux liaisons $F_{\mathrm{i}}$ en fonction de la charge extérieure $F$ n'est pas aisé sans faire les hypothèses de raideur infinie et de liaisons simplifiées (voir Fig. 3). Chacune des charges $F_{\mathrm{i}}$ dépend de la résultante des charges extérieures $F$, des raideurs du carter et de l'arbre, de la raideur globale de chacune des liaisons, et des défauts géométriques (coaxialité, perpendicularité,...). Cependant, pour des liaisons par éléments roulants, la raideur globale de celles-ci n'est pas constante (présence de contacts). Elle dépend de la charge $F_{\mathrm{i}}$, du nombre d'éléments roulants, du jeu, de la géométrie interne, des matériaux, et des défauts géométriques. La résolution du problème devra être itérative. Toute la difficulté est issue de la non-linéarité de comportement aux contacts. Dans notre cas, nous allons nous intéresser à la modélisation des contacts dans les roulements à pistes intégrées, afin d'en estimer les pressions de contact. Les pressions de contact seront calculées par la théorie de Hertz. Dans le cadre des mécaniques étudiées, nous pouvons négliger les efforts dynamiques et les forces de frottements qui sont faibles vis-à-vis des charges appliquées. Si les roulements sont soumis à de grandes vitesses de rotation et faiblement chargés, le rapport entre la force centrifuge et la force de contact appliquée sur l'élément roulant le plus chargé est supérieur à $10 \%$. Cette configuration, conduit à prendre en compte les effets dynamiques appliqués aux éléments roulants. Dans la suite du paragraphe, trois méthodes aptes à répondre à cette problématique sont présentées. L'exemple d'un roulement à billes servira d'illustration à l'application de ces méthodes.

\subsection{Méthode analytique}

La méthode analytique est la première méthode qui a été développée pour une modélisation fine des montages de roulements. Elle est utilisée dans la plupart des programmes des fabricants de roulements. L'approche

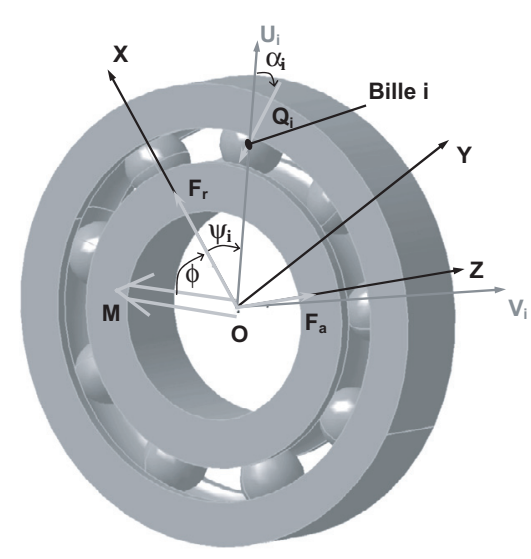

Fig. 4. Représentation des efforts extérieurs appliqués sur la bague intérieure d'un roulement à billes.

analytique du problème impose un certain nombre d'hypothèses simplificatrices sur les déformations des pièces. L'arbre est modélisé par une poutre déformable à sections variables. Le carter est supposé rigide. Seules les déformations locales au niveau des contacts (éléments roulants/pistes) sont prises en compte dans le comportement des roulements. Les équations pour la résolution du problème sont :

- pour l'arbre, les équations de la statique et la loi de comportement (modélisation 1D),

- pour les roulements, les équations de la statique, la loi de contact de Hertz et la relation entre le déplacement local de contact et le déplacement relatif entre la piste intérieure et la piste extérieure.

Les équations de l'arbre sont classiques, par contre les équations des roulements sont issues d'un couplage contact, déplacement. Afin d'illustrer ce couplage, les équations d'un roulement à billes sont détaillées cidessous. En effectuant l'équilibre de la bague intérieure du roulement (voir Fig. 4), les équations suivantes sont obtenues :

$$
\begin{gathered}
F_{\mathrm{r}}-\sum_{i=1}^{i=Z} Q_{i} \cos \left(\psi_{i}\right)=0 \\
F_{\mathrm{a}}-\sum_{i=1}^{i=Z} Q_{i} \sin \left(\alpha_{i}\right)=0 \\
M_{-} \frac{1}{2} d_{\mathrm{m}} \sum_{i=1}^{i=Z} Q_{i} \sin \left(\alpha_{i}\right) \cos \left(\psi_{i}+\varphi-\frac{\pi}{2}\right)=0
\end{gathered}
$$

En utilisant successivement la théorie de Hertz, pour les contacts entre la piste intérieure et la bille $i$, et entre la bille $i$ et la piste extérieure, la relation suivante est déduite :

$$
Q_{i}^{=} K^{\mathrm{h}} \cdot\left(\delta_{i}\right)^{n}
$$

La raideur globale de contact $K^{\mathrm{h}}$ dépend de la géométrie interne du roulement décrite sur la figure 5 (rayons de courbure). La relation entre le déplacement local du 


$$
\begin{gathered}
\sin \alpha_{\mathrm{i}}=\frac{\delta_{\mathrm{a}}+\frac{1}{2} d_{\mathrm{mi}} \theta \cos \left(\psi_{i}+\varphi-\frac{\pi}{2}\right)}{\sqrt{\left(r_{\mathrm{i}}+r_{\mathrm{e}}-D-J+\delta_{\mathrm{r}} \cos \psi_{i}\right)^{2}+\left(\delta_{\mathrm{a}}+\frac{1}{2} d_{\mathrm{mi}} \theta \cos \left(\psi_{i}+\varphi-\frac{\pi}{2}\right)\right)^{2}}} \\
\delta_{i}=\sqrt{\left(r_{\mathrm{i}}+r_{\mathrm{e}}-D-J+\delta_{r} \cos \psi_{i}\right)^{2}+\left(\delta_{\mathrm{a}}+\frac{1}{2} d_{\mathrm{mi}} \theta \cos \left(\psi_{i}+\varphi-\frac{\pi}{2}\right)\right)^{2}}-\left(r_{\mathrm{i}}+r_{\mathrm{e}}-D\right)
\end{gathered}
$$

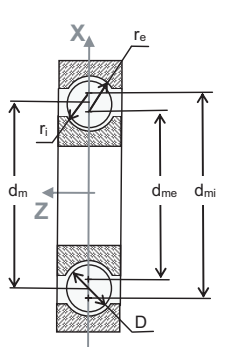

Fig. 5. Géométrie interne d'un roulement à billes.

contact de la bille $i$ et le déplacement relatif global entre la piste intérieure et la piste extérieure est la suivante :

$$
\text { voir équation (5) ci-dessus }
$$

avec :

$$
J=\left(d_{\mathrm{me}}+2 r_{\mathrm{e}}\right)-\left(d_{\mathrm{mi}}+2 r_{\mathrm{i}}\right)-2 D
$$

La relation (5) est obtenue en calculant la distance entre le point $A_{i}$ et le point $B_{s} i$ avant et après déplacement des pistes (voir Fig. 6). Cette relation permet de faire le lien entre les déplacements relatifs globaux $\left(\delta_{r}, \delta_{\mathrm{a}}\right.$, et $\theta$ entre la piste intérieure et la piste extérieure) et les déplacements locaux de contact $\left(\delta_{i}\right)$ de chaque bille $i$. Le jeu diamétral $J$ est relié à la géométrie des pistes et celles des éléments roulants par l'équation (6). L'angle de contact $\alpha_{i}$ de la bille $i$ est donné par l'orientation de la droite de contact $\left(A_{i} B_{i}\right)$ par rapport à l'axe $U_{i}$ mesuré dans le plan $\left(U_{i} Z\right)$. La relation déduite est la suivante :

$$
\text { voir équation (7) ci-dessus }
$$

La résolution globale du problème est effectuée en utilisant la méthode itérative de Newton-Raphson.

Cette méthode analytique est rapide mais ne prend pas en compte toutes les déformations présentes dans le mécanisme (déformation carter, déformation des pistes de roulement). Elle ne peut pas être directement appliquée dans les mécanismes aéronautiques étudiés. Cependant, les méthodes hybrides, présentées dans la suite de l'article, utilisent en partie des équations issues de cette méthode analytique pour modéliser le comportement local au niveau du roulement ou des éléments roulants.

\subsection{Méthode hybride E.F. et analytique utilisant les codes de contacts}

La plupart des logiciels d'éléments-finis sont capables aujourd'hui de gérer le contact entre pièces. Il existe plusieurs méthodes, les plus utilisées dans les codes de calculs industriels sont les méthodes des multiplicateurs de

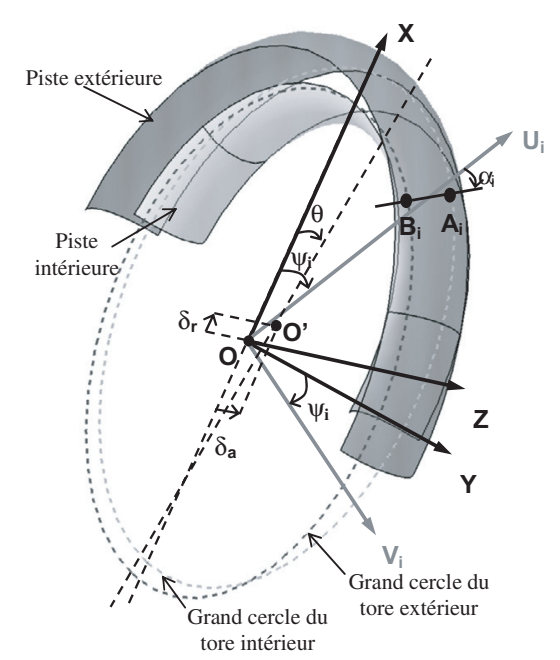

Fig. 6. Déplacements relatifs entre la bague intérieure et la bague extérieure.

Lagrange, du lagrangien augmenté et par pénalisation. Le contact, dans ces méthodes, est modélisé par une loi linéaire et le calcul est effectué avec les hypothèses des petites perturbations. Pour obtenir des bons résultats avec ce type de modélisation, il est nécessaire d'effectuer un maillage fin et symétrique des zones de contact. Avec les moyens informatiques actuels, une telle modélisation fine ne peut pas être envisagée pour des mécanismes ayant plusieurs roulements (cas d'une boîte de transmission). Pour pallier à ce problème, nous avons effectué une optimisation du maillage des roulements afin de trouver le meilleur compromis entre la qualité des résultats recherchés et le temps de calcul. Le maillage optimisé d'un roulement à billes est présenté sur la figure 7. L'optimisation du maillage des roulements a été basée sur la minimisation de l'erreur de la raideur globale du palier dans le voisinage des charges appliquées, la raideur cible étant celle obtenue par la méthode analytique décrite dans la section 3.2. Dans la modélisation par éléments-finis, deux conditions de contact ont été introduites pour chaque élément roulant des roulements à pistes intégrées : une condition de contact entre l'élément roulant et la piste intérieure et une autre entre l'élément roulant et la piste extérieure. Pour un roulement à billes avec 9000 nœuds et 200 degrés de liberté de contact, de bons résultats en efforts et déplacements ont été obtenus. Par contre, les résultats sur les pressions de contact et sur les angles de contact (pour les éléments roulants sphériques) ne peuvent être exploités (trop peu d'éléments dans la zone de contact). 


$$
\delta_{i}=\sqrt{\left(r_{\mathrm{i}}+r_{\mathrm{e}}-D-J+\delta_{\mathrm{r}}^{\text {def }}+\delta_{\mathrm{r}} \cos \psi_{i}\right)^{2}+\left(\delta_{\mathrm{a}}^{\mathrm{def}}+\delta_{\mathrm{a}}+\frac{1}{2} d_{\mathrm{mi}} \theta \cos \left(\psi_{i}+\varphi-\frac{\pi}{2}\right)\right)^{2}}-\left(r_{\mathrm{i}}+r_{\mathrm{e}}-D\right)
$$
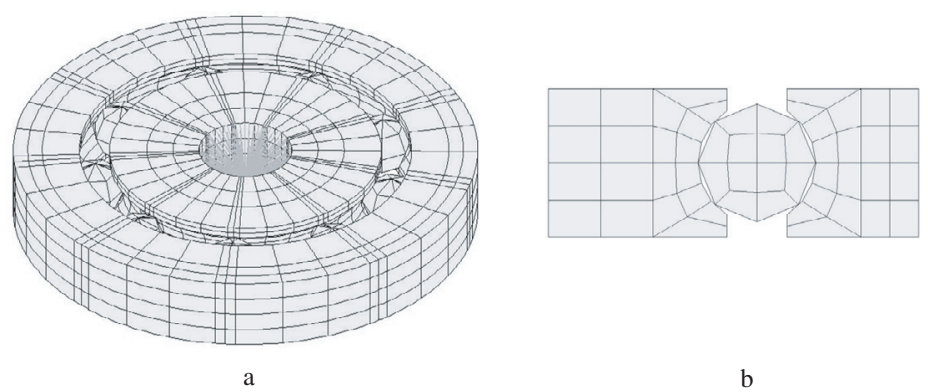

Fig. 7. (a) Maillage optimisé d'un roulement à billes en vue 3D; (b) maillage optimisé d'un roulement à billes vue en coupe.

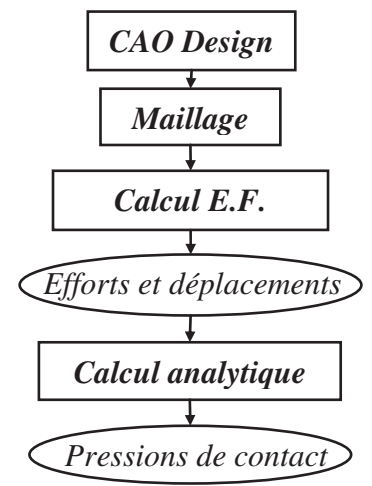

Fig. 8. Organigramme décrivant la méthode par code E.F. de contact.

La démarche développée pour calculer un mécanisme complet est décrite par l'organigramme de la figure 8. Elle consiste à effectuer le calcul en éléments-finis du mécanisme avec un maillage optimisé des roulements à pistes intégrées, de récupérer les efforts et les déplacements de chaque piste, et de calculer les pressions de contact ainsi que les angles de contact pour les éléments roulants sphériques par une méthode analytique. Le maillage d'une boîte de transmission principale d'hélicoptère est représenté sur la figure 9. La méthode analytique mise en place s'inspire de celle décrite dans la section 3.2 pour le calcul d'un roulement seul. La méthode analytique intègre la déformation des pistes et le partage des charges issues du calcul par éléments-finis. Les valeurs récupérées dans le calcul éléments-finis sont les efforts globaux $\left(F_{\mathrm{r}}, F_{\mathrm{a}}\right.$, et $\left.M\right)$, l'angle de déphasage $\phi$ (entre $M$ et $F_{\mathrm{r}}$ ), et le champ de déplacements des pistes de chaque roulement. Nous nous ramenons à un calcul analytique de chaque roulement individuellement. Le champ de déplacements de chaque piste est traité de façon à soustraire les déplacements de corps rigide afin de n'avoir que des déplacements issus de la déformation. Ce dernier est reporté sur la piste intérieure, puis caractérisé par des paramètres d'une série de Fourier en utilisant une méthode des moindres carrés. Ces paramètres définissent la déformation équivalente des pistes, et permettent de prendre en compte l'effet de la déformation globale du mécanisme modélisé. Dans le cas d'un roulement à billes, ces paramètres sont introduits dans les relations (5) et (7) de façon à bruiter la géométrie nominale. Par exemple, la relation (5) s'écrit comme suit :

$$
\text { voir équation (8) ci-dessus }
$$

avec

$$
\begin{aligned}
\delta_{\mathrm{r}}^{\mathrm{def}}= & a_{0}^{\mathrm{r}}+\left(a_{2}^{\mathrm{r}} \cos 2 \psi_{i}+b_{2}^{\mathrm{r}} \sin 2 \psi_{i}\right) \\
& +\left(a_{3}^{\mathrm{r}} \cos 3 \psi_{i}+b_{3}^{\mathrm{r}} \sin 3 \psi_{i}\right) \\
\delta_{\mathrm{a}}^{\mathrm{def}}= & \left(a_{1}^{\mathrm{a}} \cos \psi_{i}+b_{1}^{\mathrm{a}} \sin \psi_{i}\right)+\left(a_{2}^{\mathrm{r}} \cos 2 \psi_{i}+b_{2}^{\mathrm{r}} \sin 2 \psi_{i}\right)
\end{aligned}
$$

Avec les pulsations choisies, seulement les premiers modes de déformations sont récupérés. Il n'est pas nécessaire d'aller plus loin dans l'identification du champ de déplacements. Les charges sur chaque élément roulant s'obtiennent en résolvant simultanément les équations (1)-(3) (utilisation de la méthode de NewtonRaphson) avec les paramètres et les valeurs récupérés dans le calcul par éléments-finis. Le champ de pressions est calculé à partir de la charge sur l'élément roulant en utilisant la théorie de Hertz avec les rayons de courbure nominaux.

La méthodologie développée permet d'obtenir les pressions de contact dans les roulements à pistes intégrées en tenant compte des déformations de toutes les pièces du mécanisme. Elle s'implante bien dans un environnement CAO. Le mécanisme est conçu sous le logiciel CATIA V5. Toutes les pièces sauf les roulements à pistes intégrées sont maillées puis exportées dans le logiciel élémentsfinis SAMCEF. Pour les roulements à pistes intégrées, le maillage ainsi que les conditions de contact sont créés automatiquement sous SAMCEF à partir des données géométriques par un programme dédié. De plus, les temps de calcul ne sont pas excessifs (environ 3 h pour une boîte de transmission à 4 étages de réduction). Un programme a été développé pour récupérer les résultats obtenus par les éléments-finis et pour calculer les pressions de contact. 


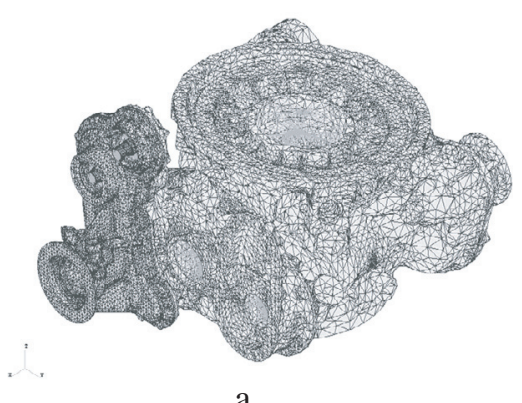

a

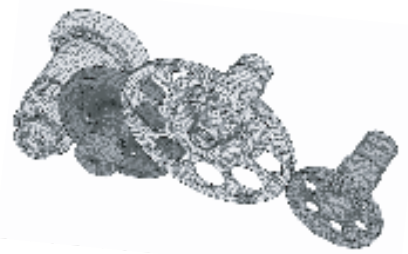

b

Fig. 9. Maillage du carter d'une transmission d'hélicoptère; (b) maillage d'une partie des pièces à l'intérieur du carter.
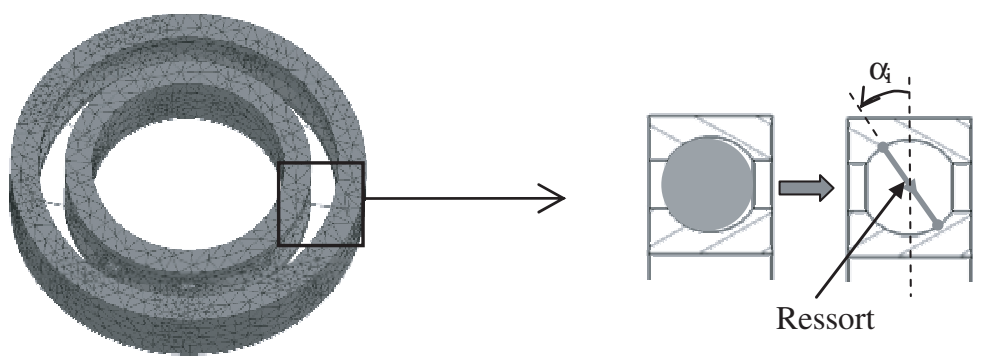

Fig. 10. Maillage d'un roulement à billes avec des ressorts.

\subsection{Méthode hybride E.F. et analytique utilisant des éléments de substitution}

La modélisation du mécanisme est réalisée par les éléments-finis sans condition de contact. Ces derniers sont remplacés par des éléments de substitution comme illustrés sur la figure 10. Cette méthode est inspirée des travaux menés par Bourdon et al. [11]. Dans cet article, les contacts entre les éléments roulants et les pistes sont modélisés par des poutres non-linéaires accrochées aux pistes. La résolution est effectuée en linéaire d'une manière itérative.

Contrairement à Bourdon et al., la méthode proposée remplace les contacts entre les éléments roulants et les pistes par des ressorts linéaires orientés comme décrit sur la figure 10. La méthode est détaillée dans l'organigramme de la figure 11. À chaque pas d'itération, le calcul par les éléments-finis du mécanisme, équipé des éléments de substitution, est effectué. Les raideurs ainsi que l'orientation des ressorts sont recalculés à la fin de chaque itération à partir des déplacements relatifs des zones d'accrochages de chaque élément de substitution, et des efforts subis par ces derniers. La position d'équilibre du mécanisme est atteinte quand la différence relative entre la somme des raideurs des ressorts du pas $p+1$ et du pas $p$ est inférieure à $0,1 \%$. La méthode analytique mise en place pour le calcul des raideurs et des orientations utilise des équations similaires (dans le cas d'un roulement à billes) à celles de la méthode décrite dans la section 3.2. Les données d'entrée du calcul analytique, à chaque pas $p$ et pour chaque élément de substitution $i$, sont le déplacement relatif $U_{\Delta i}^{p}$ entre les zones d'accrochages et l'effort appliqué $Q_{i}^{p}$. À partir de ces données, le déplacement réel local de contact et l'orientation réelle de l'effort sont calculés.

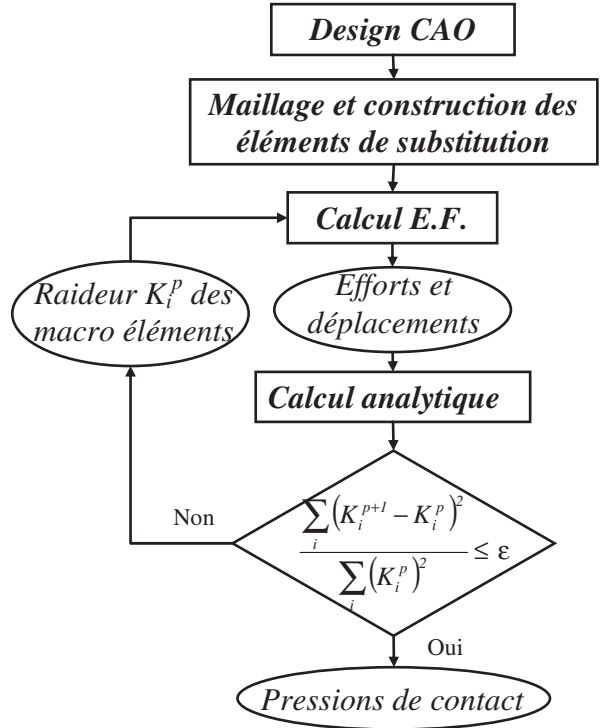

Fig. 11. Organigramme décrivant la méthode par éléments de substitution.

Dans le cas d'un roulement à billes, le déplacement local de contact $\delta_{i}^{p}$ s'obtient directement à partir de l'effort appliqué $Q_{i}^{p}$ et la raideur de contact de Hertz en inversant la relation (5). L'angle de contact $\alpha_{i}^{p}$ est plus délicat à obtenir. Il est nécessaire de connaître la décomposition du déplacement en radial $U_{\text {ri }}^{p}$ et en axial $U_{\text {ai }}^{p}$ entre les pistes intérieure et extérieure au voisinage de l'élément roulant $i$. Pour cela, il faut résoudre l'équation suivante :

$\delta_{i}^{p}=\sqrt{\left(r_{\mathrm{i}}+r_{\mathrm{e}}-D-J+U_{\mathrm{ri}}^{p}\right)^{2}+\left(U_{\mathrm{ai}}^{p}\right)^{2}}-\left(r_{\mathrm{i}}+r_{\mathrm{e}}-D\right)$ 


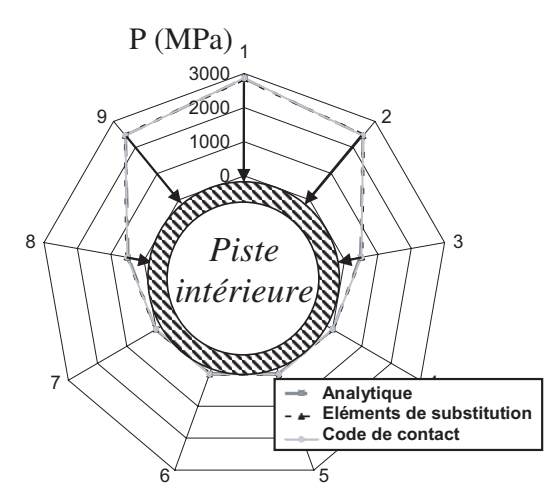

a

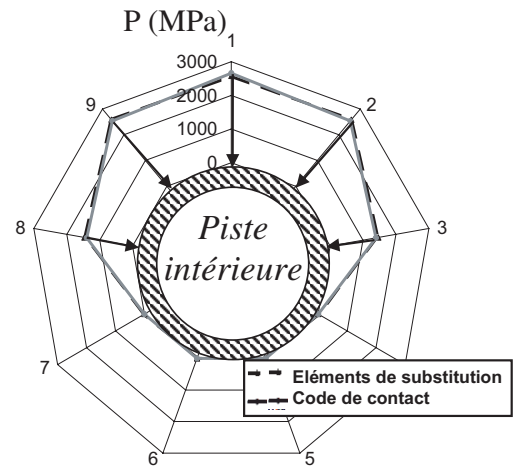

b

Fig. 12. (a) Graphique des répartitions des pressions en considérant la structure rigide; (b) graphique des répartitions des pressions en considérant la structure élastique.

Elle ne suffit pas pour déterminer le déplacement radial et axial. Il est nécessaire d'introduire une relation supplémentaire. Cette dernière est obtenue à partir du rapport entre les déplacements axial $U_{\mathrm{ai}}^{* p}$ et radial $U_{\mathrm{ri}}^{* p}$ issus du déplacement relatif $U_{\Delta i}^{p}$ de l'élément de substitution $i . U_{\Delta i}^{p}$ est issu du calcul par éléments-finis au pas $p$. La relation s'écrit comme suit :

$$
\frac{U_{\mathrm{ai}}^{* p}}{U_{\mathrm{ri}}^{* p}}=\frac{U_{\mathrm{ai}}^{p}}{U_{\mathrm{ri}}^{p}}
$$

En résolvant le système constitué des équations (11) et (12) deux couples de solutions $\left(U_{\mathrm{ri}}^{p}, U_{\mathrm{ai}}^{p}\right)$ sont trouvés. Nous choisissons la solution la plus proche du couple $\left(U_{\mathrm{ri}}^{* p}, U_{\mathrm{ai}}^{* p}\right)$ pour respecter la continuité du déplacement. L'angle de contact $\alpha_{i}^{p}$ est donné par l'équation suivante :

$$
\sin \alpha_{\mathrm{i}}=\frac{U_{\mathrm{ai}}^{p}}{\sqrt{\left(r_{\mathrm{i}}+r_{\mathrm{e}}-D-J+U_{\mathrm{ri}}^{p}\right)^{2}+\left(U_{\mathrm{ai}}^{p}\right)^{2}}}
$$

La raideur équivalente de l'élément de substitution $i$, au pas $p$, s'obtient par la relation :

$$
K_{i}^{p}=\frac{Q_{i}^{p}}{\left[U_{\mathrm{ai}}^{p} \sin \alpha_{i}^{p}+U_{\mathrm{ri}}^{p} \cos \alpha_{i}^{p}\right]}
$$

Le champ de pressions est calculé, à la fin des itérations, à partir de l'effort sur l'élément de substitution en utilisant la théorie de Hertz avec les rayons de courbure nominaux.

Cette méthodologie permet d'obtenir les pressions de contact dans les roulements à pistes intégrées en tenant compte des déformations de toutes les pièces du mécanisme. Elle a l'avantage d'être implantée directement dans l'environnement CAO (CATIA V5) utilisé pour la conception. La modélisation est effectuée sous le module d'éléments-finis (GPS) de CATIA V5. La raideur ainsi que l'orientation (angle de contact) des ressorts sont pilotées par une macro VB de CATIA V5. En général, la solution converge au bout de quelques itérations. Le nombre d'itérations est inférieur à 10. Par contre, le temps de mise à jour du modèle éléments-finis par l'interface VB dans CATIA V5 pénalise la durée de calcul.

\section{Comparaison des méthodes}

Dans les sections précédentes, trois méthodes ont été proposées pour calculer les pressions entre les éléments roulants et les pistes. La méthode analytique ne permet pas d'inclure les déformations générales de la structure du mécanisme. Cette remarque rend cette méthode inutilisable pour le problème traité. La deuxième méthode est basée sur un calcul hybride associant les éléments-finis dans un premier temps et l'approche analytique dans un deuxième temps. Elle a été mise en place et testée avec succès sur des problèmes réels. La dernière méthode hybride est basée sur un calcul par éléments-finis et sur la déformation d'éléments de type ressort. Le contact est décrit avec des ressorts dont la raideur et l'orientation sont calculées par une approche analytique. Elle a donné de très bons résultats sur des ensembles mécaniques. En conclusion, les deux dernières méthodes conviennent aux impératifs fixés par l'environnement aéronautique.

Afin de comparer la justesse relative des résultats fournis, une simulation a été menée. Dans un premier temps, une structure simple est considérée rigide (roulement à 9 billes soumis à une charge radiale de $7000 \mathrm{~N}$ au droit de la bille 1). Dans ce cas, la méthode analytique peut être aussi utilisée. Cette comparaison permet de confronter les deux méthodes développées à la méthode analytique utilisée par les fabricants de roulements. Les résultats obtenus durant cette simulation sont présentés sur la figure 12a. Les valeurs obtenues par les trois méthodes sont similaires. La différence entre les pressions maximales calculées est inférieure à $1 \%$.

Dans un deuxième temps, la déformabilité de la structure est prise en compte. Les résultats obtenus durant cette simulation sont présentés sur la figure $12 \mathrm{~b}$. Les deux méthodes proposées, hors analytique, donnent des résultats semblables. La différence maximale atteinte entre les deux estimations est de $3 \%$.

Il est intéressant de noter la différence de répartition des pressions maximales sur la piste intérieure entre les deux simulations présentées. La prise en compte de la déformabilité des bagues du roulement fait diminuer de $10 \%$ la valeur de la pression sur l'élément 
roulant le plus chargé (la bille numéro 1). Ce type de calcul montre l'effet bénéfique de la déformation de la structure sur la répartition des pressions de contact. Après expérimentation, il pourrait conduire à une optimisation du dimensionnement ou à une augmentation des spécifications géométriques des pistes intégrées.

Ces deux méthodes sont incorporées dans le processus de conception des boîtes de transmission de puissance. Le modèle de CAO du mécanisme est réalisé avec le logiciel CATIA V5. La méthode hybride utilisant les éléments de substitution est implantée dans le module de calcul par éléments-finis de CATIA V5. Par contre, la méthode hybride utilisant un code de contact est externe au logiciel de CAO. Cette méthode est implantée dans le logiciel SAM$\mathrm{CEF}$, et il est, donc, nécessaire de transférer le modèle CAO de CATIA V5 à SAMCEF.

\section{Conclusion}

Dans cet article, une étude bibliographique a été proposée pour mettre en évidence les méthodes capables de déterminer les pressions de contact entre les éléments roulants et les pistes intégrées dans un mécanisme aéronautique. Deux méthodes ont été étudiées, améliorées et adaptées à l'environnement aéronautique. Une comparaison a été effectuée sur les résultats fournis et sur leur mise en ouvre dans l'environnement CAO. Cette comparaison a permis de conclure sur la similitude des résultats obtenus et sur la propriété de la méthode hybride utilisant les éléments de substitution à s'intégrer parfaitement dans le logiciel de conception. De plus, l'effet bénéfique de la déformation de la structure sur la répartition des pressions de contact a été mis en évidence. Cette étude servira de base à l'introduction des défauts géométriques dans le mécanisme afin d'en déterminer leurs influences sur la tenue en service.

\section{Références}

[1] ISO 281, Charges dynamiques de base et durée nominale

[2] R. Stribeck, Ball bearing for various loads, Trans. ASME 29 (1907) 420-463

[3] ISO 76, Charges statiques de base
[4] G. Lundberg, A. Palmgren, Dynamic Capacity of Rolling Bearings, Acta polytechnica 7, Mech. Eng. Ser. 1 (1947)

[5] A.B. Jones, A general theory for elastically constrained ball and radial roller bearings under arbitrary load and speed conditions, Trans. ASME 82 (1960) 309-320

[6] T. Harris, Rolling Bearing Analysis 4th edition, Wiley Interscience, 2001

[7] A.B. Jones, T. Harris, Analysis of a rolling element idler gear bearing having a deformable outer race structure, ASME Trans., J. Basic Eng. (1963) 273-279

[8] S. Zupan, I. Prebil, Carrying angle and carrying capacity of large single row ball bearing as a function of geometry parameters of the rolling contact and the supporting structure stiffness, Mech. Machine Theory 36 (2001) 1087-1103

[9] T. Hauswald, L. Houpert, Numerical and experimental simulations of performances of bearing system, shaft and housing, Account for global and local deformations, Presented at the SIA seminar "Fiabilité experimentale", Oct. 24, 2000, Paris, Proceedings of the Conference

[10] T. Hauswald, L. Houpert, Simulations des performances d'un système avec roulements. CNRS paper published in Phoebus 13, $2^{\mathrm{e}}$ trimestre (2000) 77-89

[11] A. Bourdon, J. Rigal, D. Play, Static rolling bering model in a C.A.D. Environment for the study of complex mechanisms: Part I and II, Rolling bearing model, ASME Trans., J. Tribology 121 (1999) 205-214, 215-224

[12] M.R. Lovel, M.M. Khonsari, R.D. Marangoni, A finiteelement analysis of the frictional forces between a cylindrical bearing element and MoS2 coated and uncoated surfaces, Wear 194 (1996) 60-70

[13] H. Zhao, Analysis of load distribution within solid and hollow roller bearings, ASME Trans., J. Tribology 120 (1998) 134-139

[14] Y. Kang, P.C. Shen, C.-C. Huang, S.-S. Shyr, Y.-P. Chang, A modification of the Jones-Harris method for deep-groove ball bearings, Tribology Int. 39 (2006) 14131420.

[15] L. Houpert, An Engineering Approach to Hertzian Contact Elasticity, part I and II, ASME J. Tribology 123 (2001) 582-588, 589-594

[16] N. Tanaka, A new calculation method of Hertz elliptical contact pressure, ASME J. Tribology 123 (2001) 887-889

[17] J.F. Antoine, C. Visa, C. Sauvey, G. Abba, Analytical solution for Hertzian contact problems, ASME J. Tribology 128 (2006) 660-664 